\title{
DESENVOLVIMENTO DE UMA ESTRATÉGIA DE CONTENT MARKETING PARA REDES SOCIAIS: O CASO DA ESCOLA SUPERIOR DE CIÊNCIAS EMPRESARIAIS DO INSTITUTO POLITÉCNICO DE VIANA DO CASTELO
}

\author{
Cairrão, Álvaro ${ }^{1}$ \\ Instituto Politécnico de Viana do Castelo, Portugal \\ acairrao@esce.ipvc.pt \\ Garcia, Jorge ${ }^{2}$ \\ Instituto Politécnico de Viana do Castelo, Portugal \\ jorgegarcia@esce.ipvc.pt \\ Seixas, Joana ${ }^{3}$ \\ Instituto Politécnico de Viana do Castelo, Portugal \\ pjoana@ipvc.pt
}

Material original autorizado para su primera publicación en la revista académica REDMARKA. Revista Digital de Marketing Aplicado.

https://doi.org/10.17979/redma.2018.01.021.4845

Recibido: 27 enero 2018

Aceptado: 8 agosto 2018

\footnotetext{
${ }^{1}$ Licenciado em Relações Públicas pela Universidade Fernando Pessoa - Porto, licenciado em Publicidade e Relações Públicas, Mestre e Doutor em Ciências da Comunicação pela Universidade Autónoma de Barcelona. Bolseiro de Doutoramento da FCT. A realizar o Pós-Doutoramento no ICS da Universidade do Minho. Experiência de docência desde 1995 até 2018: Universidade Fernando Pessoa - Porto, Instituto Superior Miguel Torga - Coimbra, Instituto Politécnico de Viana do Castelo, Universidade de Trás-osMontes e Alto Douro - Vila Real e Universidade Portucalense - Porto. Porto, Portugal.

${ }^{2}$ Professor no Instituto Politécnico de Viana do Castelo (IPVC) desde 2005/2006. Doutorado em Engenharia Informática pela Faculdade de Engenharia da Universidade do Porto. Mestre em Engenharia Informática pela Faculdade de Engenharia da Universidade do Porto e licenciado em Ciência de Computadores pela Faculdade de Ciências da Universidade do Porto. Porto, Portugal.

${ }^{3}$ Mestre em Marketing pela Escola Superior de Ciências Empresariais e licenciada em Gestão Artística e Cultural pela Escola Superior de Educação (IPVC). Estudou "Animation as a Learning Tool" na VIA University College em Viborg, Dinamarca e especializou-se em Desenvolvimento de produtos multimédia pela Escola Superior de Tecnologia e Gestão do IPVC. Desde 2014 que desempenha funções como Produtora de Conteúdos no Jornal o Valenciano. Freelancer na produção trabalhos de fotografia e vídeo para media, organização de eventos e gestão de redes sociais e web. Valença, Portugal.
} 


\section{Resumen}

As redes sociais são cada vez mais utilizadas pelas empresas e marcas, como um dos principais meios de divulgação de produtos e serviços, devido ao crescimento que estas plataformas tiveram nos últimos anos. Como tal, as instituições de Ensino Superior não são exceção e utilizam os conteúdos publicados nas redes sociais como forma de divulgação da própria instituição e da sua oferta formativa.

Especificamente, o content marketing tem vindo a tornar-se numa das estratégias mais utilizadas como forma de aumentar o engagement, e captar novos seguidores nas redes sociais.

O objetivo principal consiste na definição e implementação de uma estratégia de content marketing para as redes sociais Facebook e Instagram, através do desenvolvimento de um caso de estudo na Escola Superior de Ciências Empresariais (ESCE) do Instituto Politécnico de Viana do Castelo, que consiga gerar um aumento da notoriedade da escola e um consequente aumento do número de alunos.

Pretende-se ainda criar maior identificação dos alunos, melhorar o engagement das redes sociais com os seus seguidores e obter mais interação por parte de utilizadores que não interagem normalmente com os perfis das redes sociais da ESCE.

Para alcançar os objetivos propostos, a fundamentação teórica determina as bases necessárias para a definição da estratégia a ser desenvolvida, bem como legitima os seus resultados que, sustentados no estudo de caso, enquanto estratégia de pesquisa, é adequado quando se pretende investigar o como e o porquê de um conjunto de eventos contemporâneos, e por isso requer múltiplos métodos e fontes para explorar, descrever e explicar o fenómeno no seu contexto. 
Espera-se que a aplicação da estratégia de content marketing desenvolvida, tenha um aumento significativo do engagement nas redes sociais, bem como na interação por parte de utilizadores que não interagem normalmente com os perfis das redes sociais da ESCE.

Palavras chave: Social Media, Content Marketing, Estudo de caso, Ensino Superior

\section{Abstract}

Social networks are increasingly being used by companies and brands as one of the main means of disseminating products and services, concerning to the exponential growth that these platforms have had in the last few years.

Therefore, Higher Education Institutions aren't exception and are also using the contents published on social networks as a way of advertising the institution itself and its training offer.

Content marketing has increasingly become one of the most used strategies by companies and brands to increase engagement and attract new followers on their social networks.

The main goal is to define and implement a content marketing strategy for social networks as Facebook and Instagram, through the development of a case study of Escola Superior de Ciências Empresariais (ESCE) of the Polytechnic Institute of Viana do Castelo, that can generate an increase in the school's awareness and following increase the number of new students.

This project also aims to create greater identification from students, improve the engagement of the social networks with their followers and to get more interaction from users who don't usually interact with ESCE's social network profiles.

In order to achieve the proposed goals, the theoretical basis defines the bases for the definition of the strategy to be developed, as well as legitimizes its results, which, based on the case study, as a research strategy, is adequate when 
investigating the how and the why of a set of contemporary events, and therefore requires multiple methods and sources to explore, describe and explain the phenomenon in its own context.

It is expected that the implementation of the content marketing strategy developed will have a significant increase in engagement in social networks as well as in the interaction by users who do not normally interact with ESCE's social network profiles.

Keywords: Social Media, Content Marketing, Case Study, Higher Education.

\section{FUNDAMENTAÇÃO TEÓRICA}

Os conceitos tradicionais de comunicação com o cliente estão a ser substituídos pela partilha de conteúdo com informação valorizada pelo consumidor. Segundo Pulizzi e Handley (2015), 96\% das empresas com estratégias B2C (Business to Consumer) utilizam o content marketing como uma das principais ferramentas da sua estratégia, acrescentando que o fundamental é "mover" o consumidor, fazer com que ele se sinta afetado de uma forma positiva e assim criar um envolvimento entre ambas as partes, atribuindo ao utilizador um papel importante e útil.

O mesmo se aplica às universidades e outras instituições de ensino superior que pretendam captar, manter e fidelizar alunos. A criação de uma comunidade, através do content marketing, é o ponto de partida para que uma escola possa atrair, reter e criar engagement a partir do momento em que os utilizadores se tornam candidatos até o momento em que se tornam alunos.

A Escola Superior de Ciências Empresariais (ESCE) do Instituto Politécnico de Viana do Castelo (IPVC), está atualmente presente nas redes sociais Facebook e Instagram. No entanto, apesar de ter um número considerável de seguidores, consideramos que o engagement com os seus seguidores é relativamente reduzido. Apenas em algumas situações excecionais, como eventos académicos e científicos existe uma maior interação com os seguidores, que por 
consequência traduz-se num ligeiro aumento de seguidores. Isso deve-se, em parte, à inexistência de uma estratégia de web marketing estrategicamente delineada a pensar nos utilizadores.

O content marketing é uma técnica de marketing de criação e distribuição de conteúdo valioso, relevante e consistente para atrair um segmento de público bem definido, com o objetivo de obter ações rentáveis por parte dos clientes. Este projeto combina a criação de uma metodologia para a implementação e análise da estratégia de content marketing, aplicada a uma instituição de ensino superior, o que permitirá uma avaliação mais completa, comparativamente a outros estudos realizados.

Carvalho (2014), num estudo sobre os fatores que geram o envolvimento dos utilizadores, concluiu que os motivos que levam as pessoas a colocarem "gosto", partilharem e comentarem conteúdos no Facebook, está relacionado com o facto de se identificarem com a mensagem que estes transmitem. No mesmo estudo refere-se ainda que, para criar envolvimento com os utilizadores de forma efetiva, não é suficiente partilhar conteúdos, mas gerar conteúdos de qualidade, sendo que os conteúdos que os utilizadores mais reagem são as imagens, imagens/textos, e os vídeos.

Uma das formas de captar novos alunos é através duma comunicação de proximidade com os mesmos e isso pode conseguir-se através das redes sociais da escola, pois é a faixa etária do grande grupo potencial que mais utiliza as redes sociais. 95\% dos adolescentes portugueses, acima dos 15 anos, estão presentes nas redes sociais (Simões, 2011).

O objetivo principal deste projeto é a definição e implementação de uma estratégia de content marketing para as redes sociais da Escola Superior de Ciências Empresariais do Instituto Politécnico de Viana do Castelo, como forma de aumentar a notoriedade, a brand awareness da marca ESCE e a captação de novos alunos. 
Para além do objetivo geral do projeto, pretende-se atingir os seguintes objetivos secundários, decorrentes da implementação da estratégia de content marketing, que posteriormente será analisada e monitorizada: a) Criar maior identificação dos alunos com a ESCE; b) Melhorar o engagement das redes sociais da ESCE com os seus seguidores; c) Obter mais interação por parte de utilizadores que não interagem normalmente com os perfis das redes sociais da ESCE.

\subsection{Marketing Digital}

O marketing digital não surge como uma substituição do marketing tradicional, mas sim como uma forma de complementar a interação com os consumidores, (Kotler, et al. 2017). O marketing digital é, como o próprio nome indica um marketing com as bases tradicionais, mas com a utilização das ferramentas digitais.

A Internet e os avanços tecnológicos permitem que a comunicação seja feita através de multiplataformas e que as instituições consigam comunicar com os seus clientes utilizando formas inovadoras. Do outro lado estão os consumidores, que cada vez mais utilizam essas mesmas plataformas para obter mais informações sobre os produtos e serviços que pretendem adquirir antes de tomar uma decisão de compra.

Para a criação de uma estratégia consolidada de marketing digital, é necessário recorrer a um conjunto de táticas que serão fundamentais para uma boa presença online e para o sucesso das empresas. Marques (2014) enumera um conjunto de táticas a ter em conta durante o processo de criação e execução de um plano de marketing digital: Blogues; E-mail Marketing; Landing Page; Mobile Marketing; Monitorização; Newsletter; PPC (Pay-per-click); SEM (Search Engine Marketing); SEO (Search Engine Optimization); Social Media; e, Website.

\subsection{Os 8 P's do Marketing Digital}


Adolpho (2012) apresenta um novo paradigma, os 8 P's do marketing digital. É um processo que funciona de forma circular, pois inicia com o consumidor e termina com o consumidor e deve ser seguido rigorosamente passo-a-passo para que a principal estratégia de marketing digital, fundamentada no grau de atividade do consumidor, seja concluída com sucesso.

As oito variáveis do marketing mix digital são:

a) Pesquisa: a criação de um produto/serviço, bem como as estratégias de marketing serão muito mais efetivas se a empresa/instituição conhecer bem as preferências dos potenciais clientes;

b) Planeamento: análise dos dados recolhidos durante a pesquisa e delinear a estratégia de marketing;

c) Produção: realização das ideias propostas no Planeamento, tendo em conta que é necessário escolher ou criar plataformas onde será feita a comunicação;

d) Publicação: o conteúdo gera a perceção do valor da marca por parte dos consumidores. Se o conteúdo for relevante para eles, vão interagir e podem até tornar uma marca viral;

e) Promoção: as campanhas de marketing devem ter utilidade para potencial cliente e não apenas apresentar um determinado produto ou serviço e que a criação de conteúdo relevante é a forma mais eficaz de oferecer uma informação útil aos consumidores. $\mathrm{O}$ consumidor gera muito mais comunicação do que a própria empresa;

f) Propagação: após um bom trabalho de SEO e na criação de conteúdo de interesse, a empresa deverá trabalhar de forma a que o conteúdo seja partilhado e crie impacto a um maior número de pessoas. Se os clientes gostarem do que viram irão partilhar o conteúdo de forma orgánica. Ainda assim, a empresa pode agir de forma a potencializar essa difusão, como pagar para impulsionar posts no Facebook, ou usar pequenas estratégias juntamente com os posts como fazer 
perguntas e pedir opinião, pois há uma possibilidade elevada das pessoas "gostarem" da publicação, comentarem e partilharem o conteúdo;

g) Personalização: comportamentos, valores, formas de ver a vida, são elementos que alteram de pessoa para pessoa e, como tal, cada consumidor tem uma perceção diferente dos produtos/serviços. Cabe às empresas fazerem uma segmentação e adaptarem-se ao comportamento de cada pessoa que, num contexto digital;

h) Precisão: capacidade para medir os resultados, tendo em conta todas as fases do processo. As métricas são o mais importante de todo o processo.

\subsection{Web 2.0 e User Generated Content}

Uma das características da Web 2.0. é o User Generated Content (UGC) que se refere a informações e materiais produzidos e publicados por utilizadores de Internet, que podem ser produzidos individualmente ou em colaboração, modificados, partilhados e apresentar-se das mais distintas formas, desde tweets no Twitter, posts no Facebook, como avaliações de consumidores (consumer reviews), publicidade, entre outras. Cada vez mais, os consumidores dependem particularmente do UGC para a tomada de decisão. É, possivelmente, o elemento mais poderoso de uma comunidade online, por ser personalizado e os utilizadores poderem exercer mais controle na criação de conteúdo do que os próprios produtores de bens e serviços (Dickey \& Lewis, 2011).

Os utilizadores confiam mais no conteúdo criado por outros utilizadores do que no conteúdo gerado e partilhado pelo produtor/marcas, pois acreditam que outros utilizadores irão partilhar experiências positivas e negativas dos produtos com intenção de divulgação completa sem qualquer tipo de interesse comercial, o que torna a avaliação dos produtos ou serviços mais realistas.

O electronic word-of-mouth (EWOM) é criado quando os consumidores geram as suas próprias informações na Internet, ou seja, qualquer estado positivo ou negativo feito por clientes reais ou antigos sobre o produto ou empresa, que está 
disponível nas mais diversas plataformas na Internet, desde fóruns, reviews, redes sociais, chats e blogs para um enorme número de pessoas e instituições, e tem uma grande influência no comportamento do consumidor, formando a intenção de compra dos consumidores e afetando o comportamento da compra.

\subsection{Social Media}

Os social media são um conjunto de aplicações na Internet que permitem a criação e partilha de conteúdos por utilizadores e permitem que os utilizadores produzam conteúdos, de forma individual ou em comunidades, de diversos formatos e para publicação e partilha nas plataformas digitais onde se encontram utilizadores que vêem, gostam, comentam, partilham diariamente esses conteúdos.

Kotler, et al. (2017) referem que os social media têm um papel fundamental na forma como se tem vindo a alterar o paradigma da comunicação e do marketing. Nesse sentido, os consumidores não prestam a mesma atenção aos conteúdos que passam nos meios de comunicações tradicionais uma vez que têm a alternativa dos conteúdos gerados por utilizadores e que estes consideram mais credíveis e apelativos.

Os social media podem agrupar em seis diferentes grupos como: Projetos coletivos, como o caso da Wikipédia; Blogs; Jogos online com multiplayers; Realidades Virtuais, como o Second Life; Comunidades de conteúdos, tais como - Youtube e as Redes sociais, desde Facebook, Instagram, LinkedIn, entre outras (Kaplan \& Haenlein, 2010).

\subsection{Marketing nas redes sociais}

O marketing nos social media consiste na criação de um relacionamento e diálogo com o público-alvo para, mais do que passar a mensagem, poder receber e trocar ideias com os consumidores. Cada vez mais, o consumidor recorre aos social media para obter mais informação sobre determinados produtos e serviços, antes da decisão de compra. Por esse motivo, o papel dos 
marketeers que acompanharão todo o processo da experiência do consumidor online têm nas suas mãos, mais do que a oportunidade de ganhar a confiança desse consumidor, gerar conhecimento e converter em vendas.

Quando incorporado nos social media, o marketing consegue transformar a perceção dos consumidores e fazer com que estes se tornem mais leais à marca. Assim, o consumidor passou a ter uma participação mais ativa, emitindo as suas opiniões diretamente nas páginas das empresas. Na realidade o Facebook contribuiu para uma nova era do marketing em que deixa de colocar as empresas a falar para os consumidores ouvirem e passa a ser o inverso, o consumidor a falar e as marcas a ouvirem.

\subsection{Social media e ensino superior}

A evolução da Internet é, provavelmente, uma das mudanças mais significativas no que diz respeito ao processo de escolha de uma universidade.

Daun-Barnett, et al, (2014) afirmam que os social media poderão alterar substancialmente a forma como as instituições criam ligação com os futuros alunos. No entanto, a grande maioria das instituições ainda está à procura de qual o conteúdo mais envolvente, nessas mesmas redes, para aumentar a notoriedade da sua marca, atrair estudantes, interagir com alunos atuais e permanecer em contacto com ex-alunos.

A universidade é, para a grande maioria dos estudantes, uma das fases mais importantes e transformadoras das suas vidas, e as escolas mais bem-sucedidas nos social media estão a utilizá-los para partilhar algumas das experiências desses estudantes, referindo que o que os potenciais alunos procuram preferencialmente informação vinda de estudantes reais, por considerarem genuíno e autêntico, ao contrário das mensagens das administrações. 


\subsection{Notoriedade da marca}

A notoriedade da marca está ligada à capacidade que os consumidores e potenciais consumidores têm de reconhecer ou lembrar uma marca, associando a um determinado produto ou serviço (Aaker, 1991). Anos mais tarde, o mesmo autor, acrescenta que a notoriedade da marca se perceciona quando a mesma já se encontra presente na mente do consumidor (Aaker, 2004).

Também Keller (2003) define a notoriedade como sendo a forma como o consumidor perceciona e reconhece a marca e, posteriormente, utiliza esse conhecimento no momento da decisão de compra. No caso do presente estudo, está ligada à forma como os potenciais consumidores percecionam a marca, através da comunicação feita nas redes sociais.

\subsection{Social Media}

Holliman e Rowley (2014) compararam recentemente diferentes estudos à procura de respostas para a questão do que é marketing de conteúdo. Os auores consideram que content marketing envolve a criação, distribuição e partilha de conteúdo relevante, adequado e atraente para envolver os clientes, adaptando ainda a definição da American Marketing Association como: O content marketing digital é a atividade associada à criação, comunicação, distribuição e troca de conteúdo digital que cria valor para os clientes, parceiros e empresa e respetivas marcas.

Recentes estudos consideram o content marketing uma forma de comunicação de marketing, na qual as marcas produzem e distribuem conteúdo aos consumidores com o objetivo principal de gerar interesse, envolver os consumidores e influenciar seu comportamento (Stephen et al., 2015).

Tendo em conta os autores, pode definir-se content marketing como sendo um conjunto de ações que tem como principal objetivo criar e promover conteúdo valioso e relevante na Internet, atraindo dessa forma o consumidor. Uma 
estratégia de marketing bem-sucedida é aquela faz com que as empresas consigam interligar-se com seus clientes através de formas novas e inovadoras.

Em 2009, os autores Pulizzi e Barrett introduziram a sigla B.E.S.T. como uma fórmula de ajuda na criação de conteúdo de qualidade e de interesse do públicoalvo:

a) Behavioral - É necessário entender como queremos que os nossos clientes se sintam, o efeito que pretendemos atingir e a reação que pretendemos provocar nos mesmos, quando partilhamos o conteúdo, e por último de que forma é que vamos medir esse comportamento:

b) Essential - O conteúdo deverá ser sempre essencial para o cliente. É também importante estabelecer quais são os elementos fundamentais na campanha de content marketing, bem como os tipos de media que devemos incluir;

c) Strategic - as estratégias de content marketing deverão estar alinhadas com os objetivos estratégicos da empresa de forma a ajudar a alcançá-los. Deverão ainda estar integrados com as outras iniciativas estratégicas.

d) Targeted - É necessário identificar exatamente o target, entender quais as suas motivações e a forma como este vê o produto ou serviço que oferecemos.

Com a aplicação desta fórmula, os autores acreditam que as empresas conseguirão dar ao cliente o que realmente lhe interessa, aproveitando para partilhar as vantagens dos seus produtos e criar um diálogo que fará com que se destaque da concorrência e consiga aumentar a fidelidade do seu target.

\subsection{Métricas}

Bayer (2012) sugere quatro tipos de métricas:

a) Consumption metrics: dados sobre o número de pessoas que consumam o conteúdo que pode ser fornecidos através do número de visualizações da página, o número de novos visitantes, downloads, etc.; 
b) Sharing Metrics: frequência com que os consumidores do conteúdo o partilham é também uma das principais métricas para medir se o consumidor achou aquele conteúdo valioso ao ponto de interagir e de querer partilhar com a sua comunidade online. É possível avaliar-se através dos "likes" e "shares" nas diversas redes sociais;

c) Lead Generation Metrics: pretende avaliar a frequência com que os consumidores de conteúdo se transformam em leads, ou seja, se mostram interessados em saber mais sobre a empresa e os seus respetivos produtos/serviços. Aqui começa a avaliação do retorno do investimento que foi feito na campanha de content. Esta avaliação poderá ser feita através de por exemplo: subscrições por email ou pedidos de versões teste de determinados produtos;

d) Sales Metrics: pretende medir-se quantos dos leads foram convertidos em vendas, ou seja, quantos dos consumidores do conteúdo se tornaram em clientes.

Usando estas métricas para avaliar uma campanha de content marketing, as empresas conseguem avaliar o ROI (return of investment) de cada uma das peças da campanha.

\subsection{Tipos de conteúdos}

No que diz respeito à forma como o conteúdo é apresentado online, McPheat (2011) considera três formatos: o textual, o visual e o áudio.

De acordo com o autor, o formato visual é o que consegue mais impacto nos consumidores, mas sublinha que o conteúdo textual tem que ser bem estruturado. No que diz respeito ao áudio, considera-o importante para a perceção do conteúdo, mas na grande maioria dos casos, surge com a conjugação com outros formatos. $O$ texto como suporte é fundamental sempre que o formato visual, só por si, não tem a capacidade de ser um conteúdo inteiramente relevante. 
Também, Adolpho (2012) destaca a importância do texto, referindo que independentemente do formato, as palavras são fundamentais na definição do conteúdo.

\section{METODOLOGIA}

O processo metodologico pode utilizar tanto abordagens quantitativas como qualitativas ou ambas combinadas no mesmo estudo, por isso se tem tornado um fenómeno cada vez mais popular em estudos na área da Gestão e Marketing. Diehl (2004) sintetiza estes dois conceitos de abordagens metodológicas da seguinte forma: i) Intrínseco: diz respeito aos estudos que partem do interesse do próprio investigador em compreender melhor algum caso particular; ii) Instrumental: refere-se a estudos de caso que visam fornecer alguma introspeção sobre um assunto, para poder aumentar o conhecimento sobre o caso em si, mas que poderá proporcionar a compreensão de algo mais vasto; iii) Coletivo: é a utilização do instrumental aplicado a vários casos em simultâneo proporcionando a comparação e um conhecimento mais aprofundado.

Yin (2010) divide os projetos de estudo de caso em quatro tipos distintos: os projetos de caso único referem-se apenas a uma entidade, enquanto que os de casos múltiplos se referem a várias entidades em simultâneo; relativamente aos estudos holísticos, o autor caracteriza como sendo um estudo focado na natureza global de uma organização, enquanto que os estudos integrados, ainda que sejam em caso único, possam ser focados uma ou mais subunidades.

Classificamos o presente estudo como um projeto de caso único integrado, por se focar na análise das redes sociais da uma única instituição - a Escola Superior de Ciências Empresariais. Pode ainda referir-se que se trata de um estudo do tipo instrumental pois não se trata apenas de um estudo do interesse do investigador, mas como um instrumento que é útil para a instituição em estudo e poderá, no futuro, ser replicado e utilizado por outras instituições com características semelhantes. 
Esta metodologia pode utilizar tanto abordagens quantitativas como qualitativas no mesmo estudo.

A utilização da análise estatística permite-nos resumir e compreender um enorme número de dados numéricos, obtidos a partir das ferramentas de analytics e insights das redes sociais em estudo, através da análise de gráficos de barras e de dispersão.

O recurso às entrevistas, como fonte de dados qualitativos, enriquecerá a investigação no momento da análise dos dados, pois permite-nos comparar e cruzar a informação obtida com a análise estatística e conseguir assim conclusões mais reais e verificar o cumprimento dos objetivos propostos.

\section{PLANEMENTO, EXECUÇÃO E AVALIAÇÃO DO ESTíMULO}

A "Essência da ESCE" é o nome dado a este projeto de content marketing para as redes sociais Facebook e Instagram. A ideia deste projeto consiste na publicação regular de fotografias que retratam a autenticidade das pessoas que, diariamente, trabalham ou estudam na ESCE. Muito mais do que apenas rostos, a "Essência da ESCE pretende partilhar imagens que captam a beleza das diferentes histórias de vida da comunidade ESCE, bem como percursos e valores, mas acima de tudo captam olhares genuínos que agregam vontades e sonhos comuns.

Esta série de fotografias agregam rostos das pessoas que constituem a comunidade académica da ESCE, desde estudantes, docentes, funcionários e eventualmente convidados, palestrantes e outros membros IPVC).

Para a formalização da criação da estratégia de content marketing a ser utilizada, foi tida em conta a fórmula B.E.S.T.:

a) Behavioral - o objetivo é criar um "orgulho ESCE" em toda a comunidade de utilizadores. Fazer com que estes se sintam identificados com as publicações e, por consequência, interajam com as publicações. A avaliação será feita através do número reações à publicação, comentários, bem como número de partilhas; 
b) Essential - De forma a beneficiar os utilizadores da melhor forma, o conteúdo foi feito diretamente com eles, de forma a haver uma maior interação e haver maior relevância e interesse para o mesmo, podendo trazer benefícios a nível pessoal e profissional, nomeadamente a notoriedade e os comentários positivos que possam surgir relativamente à publicação. $O$ tipo de estímulo escolhido foi a fotografia;

c) Strategic-Fazer com que as pessoas sintam orgulho pelo local de ensino que se encontram e, por sua vez, passem essa mensagem a possíveis novos alunos. Aliado a este projeto de content marketing existem outras estratégias offline que suportam esta mesmos objetivos, como a criação de eventos de interação da comunidade académica;

d) Targeted - O nosso target são todos os nossos utilizadores, com maior foco na comunidade académica atual e nos potenciais novos alunos. A criação de conteúdo sobre o nosso target acresce o interesse pelas publicações.

\subsection{Tipos de conteúdos a desenvolver}

Tal como acontece em alguns exemplos do projeto de referência, 'Humans of New York' (2010), também o conceito visual deste projeto conjuga fotografias a cores, com orientação horizontal, que poderão ser tipo portrait ou de meio corpo, tendo em conta as características de cada pessoa. As fotografias deverão ser tiradas todas de raíz, tendo em conta os requisitos do projeto e todas elas capturadas no ambiente e nos espaços da escola, para que haja identificação também com o local. No entanto, o local não necessita estar em total destaque para que não se perca a essência da ligação com a pessoa destacada. Dessa forma, as fotografias deverão ser tiradas com uma distância e abertura focal que permite um fundo desfocado, com baixa profundidade de campo (Figuras 1).

Como forma de criar laços mais fortes com os utilizadores, nas fotografias capturadas, as pessoas fotografadas deverão ter o olhar direto para a câmara, criando assim uma ligação mais forte (Figura 2). 
O texto descritivo que deverá acompanhar todas as imagens também deverá ser criado com uma conjugação de elementos pessoais de identificação (Figura 3) com caraterísticas mais pessoais que permitam que quem conhece consiga reconhecer e quem não conhece possa conhecer melhor e criar empatia. Para isso o texto deverá ser escrito na terceira pessoa com elementos reconhecidos e não uma auto-descrição com relato da vida pessoal (Figura 3).

Figura 1: Baixa profundidade de campo

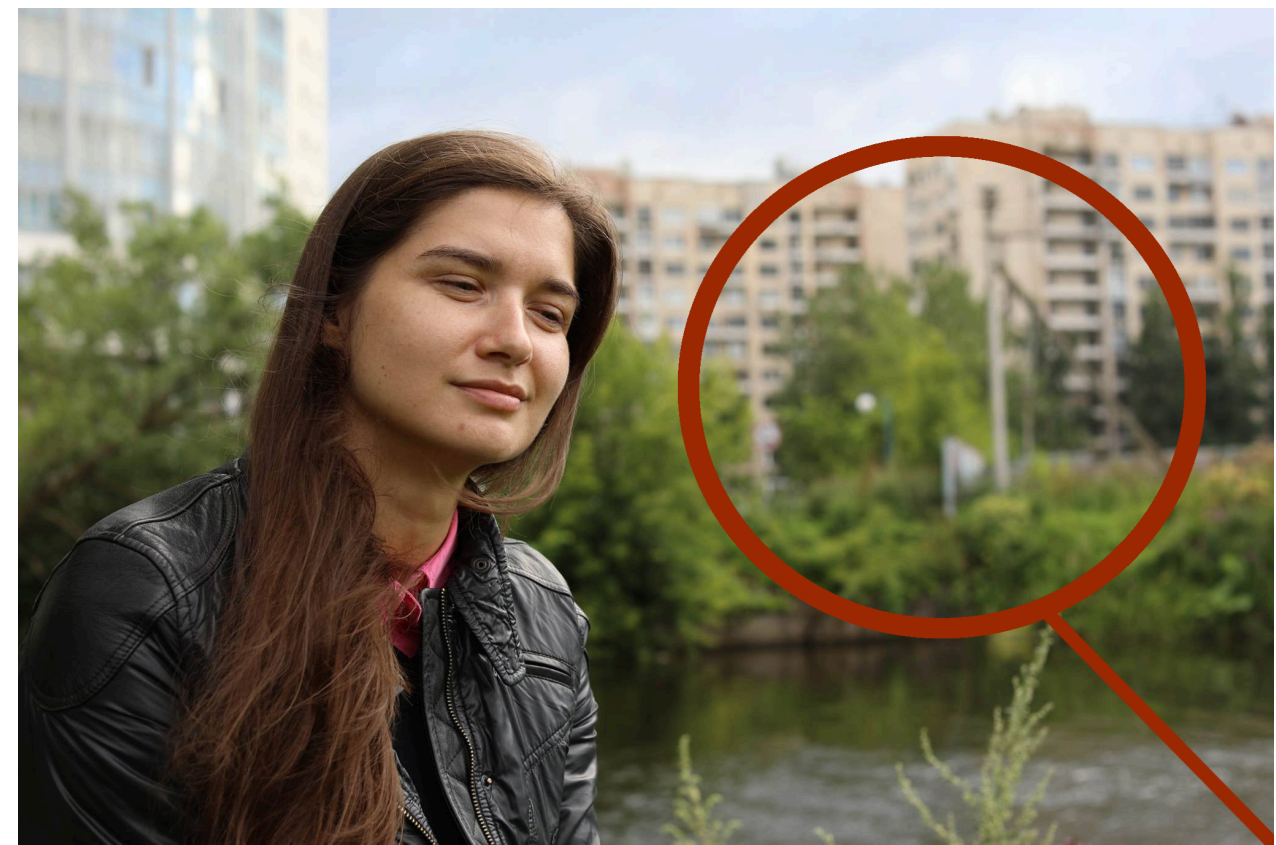

Fonte: Projeto "Humans of New York" 
Figura 2: Olhar direto para a cámara

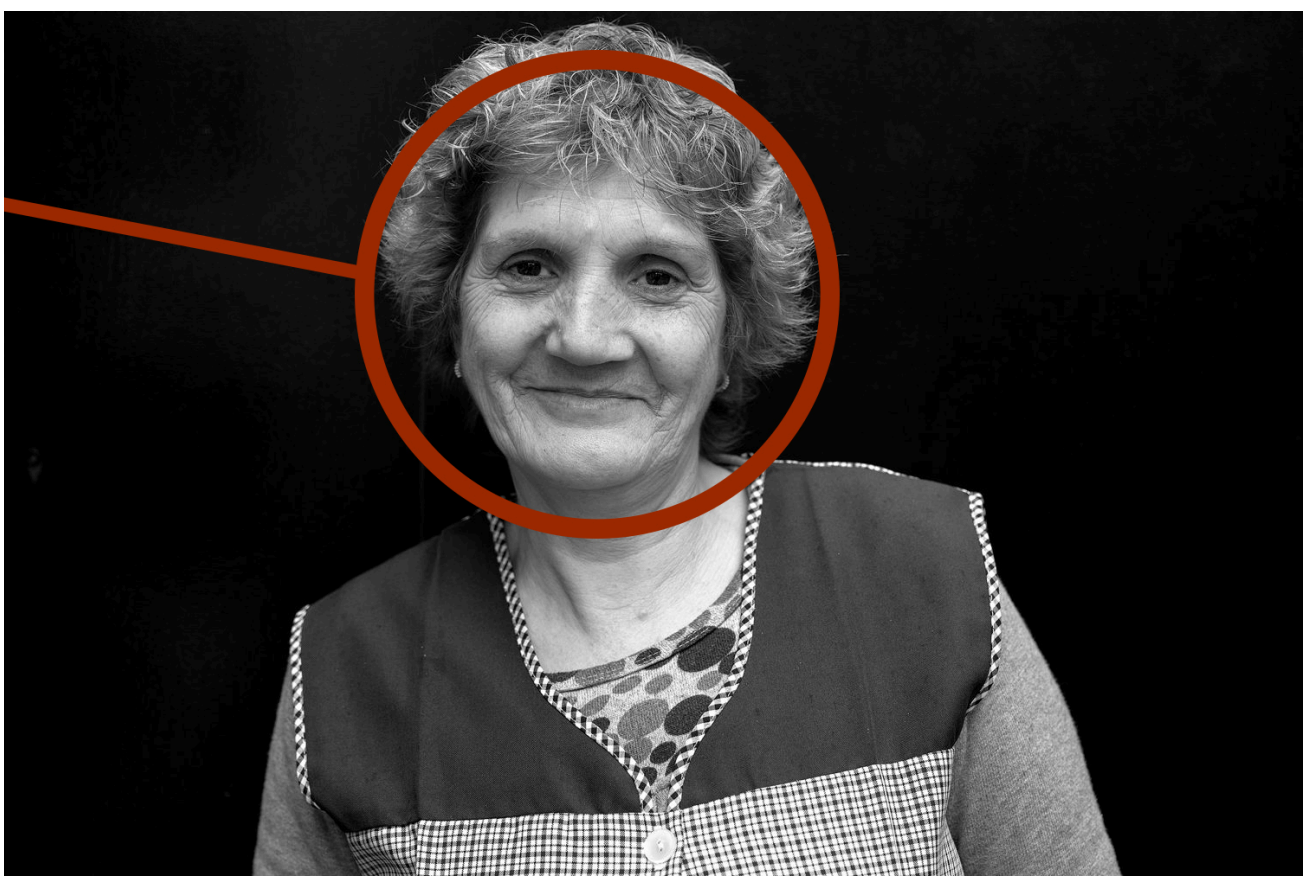

Fonte: Projeto "A pele do Porto"

Figura 3: Publicação acompanhada pelo texto descritivo

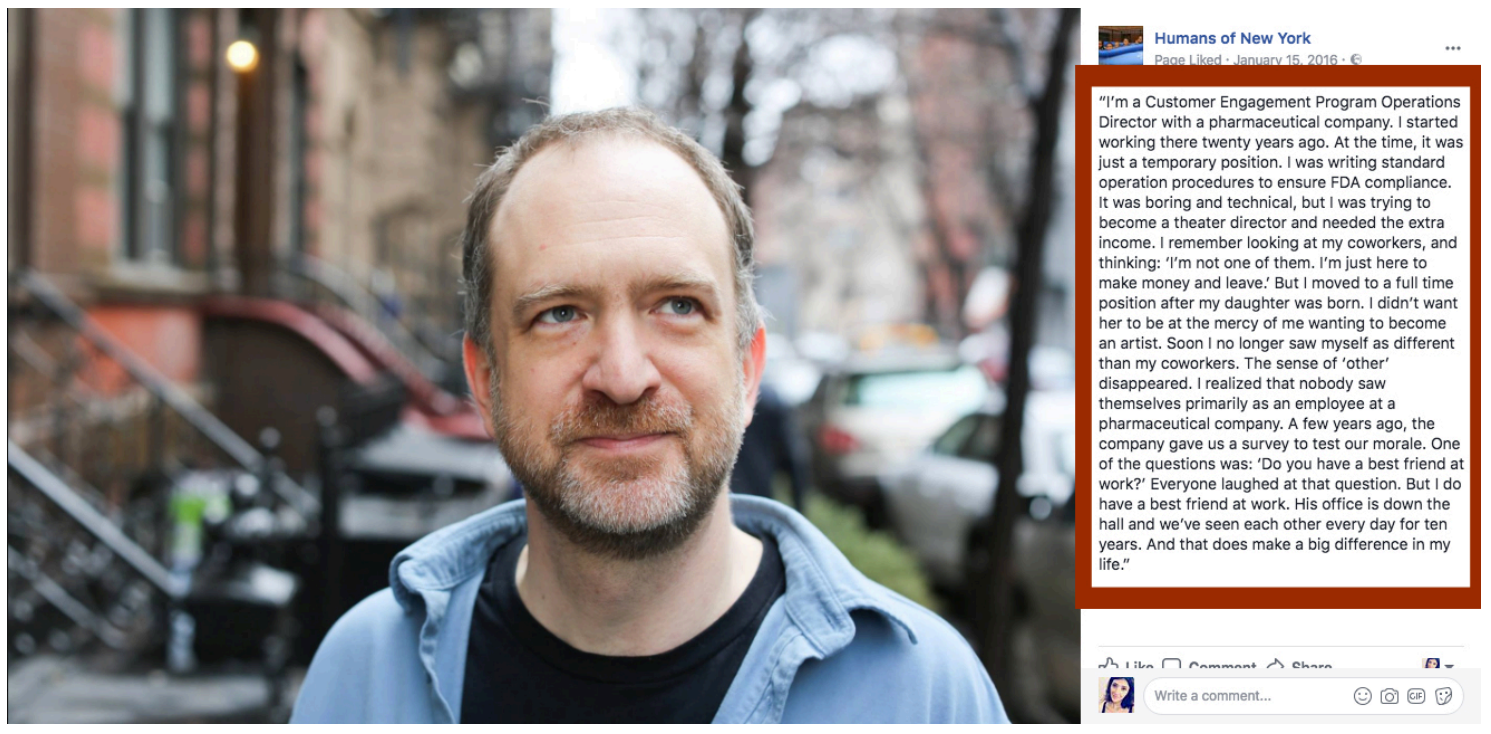

Fonte: Projeto "Humans of New York" 


\subsection{Implementação/Calendarização}

Após a definição da estratégia de content marketing, do público-alvo a quem se destina o conteúdo, o que publicar, em que formatos e plataformas, o passo seguinte foi decifrar quando fazê-lo para que os utilizadores interajam mais e se consiga o maior impacto com as publicações. Não há fórmulas e horários certos, por isso é importante que se defina um horário e uma periodicidade, e sejam feitos diferentes testes durante algum tempo, que poderá ser uma semana ou um mês, para analisar o impacto e avaliar e alterar, se necessário, em função desses resultados. Ainda assim, podem considerar-se os dados do Facebook em Portugal que revelam que depois do meio-dia, há cerca de um milhão de pessoas ligadas à rede social, sendo que o maior pico se dá ao final do dia.

Tendo em conta dados fornecidos pelas analytics da página do Facebook da ESCE e das estatísticas da conta de Instagram, diariamente às 18 horas, há uma maior concentração de seguidores online, logo esta é hora mais indicada para publicar, de forma a alcançar mais pessoas e que as mesmas interajam com a publicação.

Foram escolhidas algumas das pessoas mais populares da escola para que houvesse uma maior identificação com o conteúdo logo na fase inicial e se conseguisse captar a atenção dos utilizadores.

Durante as sessões fotográficas foi feita uma breve conversa sobre os gostos pessoais e os que as move, bem como o sentimento em relação à escola. Para alem de conseguirmos capturar olhares mais emotivos nas fotografias, permitiunos que o texto que acompanha a imagem fosse ainda mais pessoal e, dessa forma, aumentar a identificação das pessoas com o conteúdo.

Depois de reunido um conjunto de fotografias, foi feita a distribuição pelos dias, de forma a colocar alternadamente funcionários, professores e alunos.

Os locais foram também escolhidos em função das pessoas fotografadas tendo em consideração os sítios em que se sentem mais enquadradas. Na primeira 
abordagem para as fotografias foi feita uma breve apresentação do projeto para que as pessoas pudessem ter a perceção o que poderia ter o resultado final. A escolha da fotografia final teve também em conta as preferências das modelos, sempre dentro dos padrões estabelecidos. O texto ficou inteiramente da responsabilidade dos autores do projeto, sendo que as pessoas só tiveram conhecimento no momento da publicação.

Esta escolha foi feita, essencialmente por ser um período de tempo em que foi possível testar diferentes periodicidades das publicações e que se conseguiu obter diferentes variações para poderem ser contrastadas na análise de resultados.

Nos primeiros 15 dias foi testada uma periodicidade diária para testar o projeto e entender os dias com mais impacto. Depois, tendo em conta os dados recolhidos e porque havia necessidade de introduzir outro conteúdo, optou-se por partilhar entre 2 e 3 vezes por semana, respeitando os dias de mais impacto e a introdução de outros conteúdos produzidos pela escola, bem como os eventos a decorrer.

Nos meses de maio e junho, as publicações passaram a ser feitas entre 1 e 2 vezes por semana, justificando-se com a necessidade de a escola introduzir mais conteúdo promocional e, para nos mantermos fieis às estratégias das próprias redes sociais, optou-se por diminuir a quantidade de publicações do projeto e conciliar com as restantes publicações.

O início da implementação do projeto foi no dia 3 de março de 2017, com a primeira publicação nas redes sociais Facebook e Instagram. Após o teste do conceito através da realização do pré-teste anteriormente referido, o projeto encontra-se em implementação, sendo avaliado na sua totalidade antes do início do próximo ano letivo. 


\subsection{Monitorização}

Recorrendo à utilização das ferramentas de analytics do Facebook e do Instagram, vai ser monitorizado o impacto dos posts tendo em conta: a) Reações, partilhas e comentarios; b) Cliques nas fotografias; c) Visualizações da página; d) Alcance das publicações; e) Avaliar o número de posts/semana.

A monitorização será feita sempre que é publicado um novo conteúdo do projeto, bem como uma análise semanal que pretende avaliar o impacto, num contexto geral e poder haver uma adaptação das novas publicações tendo em conta os outputs obtidos.

Cada publicação de conteúdo, para além de ser publicada nos dias e horas preferenciais, será também dada relevância a outros fenómenos online e offline ligado à escola

\section{DISCUSSÃO E CONCLUSÕES}

A estratégia do projeto foi desenhada com foco na produção de conteúdo significante para o target definido. Nesse sentido encontrar um conteúdo que conseguisse ir de encontro aos objetivos do projeto e também da instituição em estudo e, em simultâneo, provocar interesse nos utilizadores de forma a fazer com que eles interagissem com o conteúdo, foi o designio estratégico do projeto.

Os consumidores privilegiam conteúdos gerados por outros utilizadores por questões maioritariamente emocionais e porque consideram mais credíveis. Desta forma, e tendo em conta as novas tendências, este projeto pretende posicionar-se através da criação de conteúdos que criem alguma relação emocional com os seguidores, e fazer com que eles queiram assim consumir mais conteúdos produzidos pela instituição.

Para este projeto optou-se pela criação de conteúdo em formato visual, por ser o conteúdo que gera mais interação por parte dos utilizadores online, sendo que este deverá sempre ser acompanhado do suporte em texto uma vez que os formatos definem melhor o conteúdo, gerando assim mais valor para o utilizador. 
As fotografias de rosto permitem que haja um reconhecimento quase imediato da pessoa e quando acompanhadas pelo texto descritivo proporciona uma identificação por parte dos utilizadores que, por sua vez, quando se sentem identificados e criam alguma relação emocional com a publicação, reagem mais rapidamente ao conteúdo com "likes", comentários e partilhas.

Até à data o projeto está em implementação, porém os resultados das métricas mensais permitem aferir que, eventualmente, no final do mesmo o engagement, a notoriedade e a captação de novos alunos será uma realidade, porém apenas após a entrada dos novos alunos no próximo ano letivo será possível confirmar.

\section{REFERÊNCIAS BIBLIOGRÁFICAS}

Aaker, D. A. (1991). Managing Brand Equity: Capitalizing on the Value of a Brand Name. New York: Free Press

Aaker, D. A. (2004). Leveraging the Corporate Brand. In California Management Review, Vol. 46, № 3.

Adolpho, C. (2012). Os 8 P's do marketing digital - O guia estratégico de marketing digital. Alfragide: Texto editores Lda.

Bayer, J. (2012). The 4 Types of Content Metrics That Matter, Disponível em: http://www.convinceandconvert.com/content-marketing-2/the-4-types-ofcontent-metrics-that-matter/ Acedido em: 09/08/2017].

Carvalho, A. (2014). Facebook infection: Marketing de Conteúdo e fatores que geram o envolvimento dos utilizadores. Lisboa: Universidade Lusófona de Humanidades e Tecnologias.

Daun-Barnett, N., et al. (2014). College counseling for admissions professionals: Improving access and retention. New York: Routledge.

Dickey, I., \& Lewis, W. (2011). An Overview of Digital Media and Advertising. In Eatin, M. S., Daugherty, T. And Burns, N.M (eds) Digital Media and advertising: 
User generated content consumption. New York: Information Science Reference $(1-31)$.

Holliman, G., \& Rowley, J. (2014). Business to business digital content marketing: marketers' perceptions of best practice. In Journal of Research in Interactive Marketing 8(4), 269- 293. http://doi.org/10.1108/JRIM-02-2014-0013

Keller, K.. (2003). Brand Synthesis: The Multidimensionality of Brand Knowledge. In Journal of Consumer Research Vol. 29, n 4.

Kotler, P., et al. (2017). Mudança do tradicional para o digital - Marketing 4.0. Lisboa: Conjuntura Atual Editora.

Marques, V. (2014). MKT digital 360. Coimbra: Actual.

McPheat, S. (2011). Developing an Internet Marketing Strategy. Disponível em: http://bookboon.com/en/developing-an-internet-marketing-strategy-ebook.

Acedido em 3 de novembro de 2017.

Pulizzi, J. \& Barrett, N. (2009). Get content, get costumers: Turn prospects into buyers with content marketing. Nova York: Mc Graw Hill.

Pulizzi, J. \& Handley, A. (2015). B2B Content Marketing: 2015 Benchmarks Budgets and Trends. Content Marketing Institute.

Simões, R. (2011) As Redes Sociais na Adolescência em Portugal - Quem Utiliza? Estudo de caso nos alunos do $2 .^{\circ}$ e $3 .^{\circ}$ Ciclo do Ensino Básico em alguns Concelhos. Universidade Nova de Lisboa

Stephen, A., et al. (2015). The Effects of Content Characteristics on Consumer Engagement with Branded Social Media Content on Facebook. In Marketing Science Institute, (15). Disponível em http://www.msi.org/reports/the-effects-ofcontent- characteristics-on-consumer-engagement-with-branded/. Acedido em 12 de novembro de 2017. 
Yin, R. (2010). Estudo de caso: planejamento e métodos. Porto Alegre: Bookman. 